\title{
21 Ethik der Prävention am Beispiel von Notfällen
}

\author{
Nico Vonneilich und Olaf von dem Knesebeck
}

- Prävention in der Notfallmedizin erscheint als ein wichtiges Handlungsfeld, insbesondere bei genauerer Betrachtung der Häufigkeitsverteilung und Risikofaktoren von medizinischen Notfällen.

- Präventive Maßnahmen in der Notfallmedizin können einen Beitrag zur Senkung von lebensbedrohlichen Komplikationen und Mortalitätsrisiken leisten, beispielsweise durch Aufklärungs- und Schulungsprogramme oder durch Früherkennung.

- Bei der Entwicklung und Umsetzung solcher Maßnahmen sollten ethische Kriterien wie Beurteilung des Nutzen- und Schadenspotenzials, Respekt der Autonomie, Gerechtigkeit, Effizienz und Legitimität berücksichtigt werden.

\subsection{Einleitung}

Vor dem Hintergrund der zunehmenden Alterung der Bevölkerung, des Wandels des Krankheitsspektrums hin zu chronisch-degenerativen und psychischen Erkrankungen sowie der veränderten Anforderungen in der Arbeitswelt wird eine effektive Gesundheitsförderung und Prävention als zunehmend wichtig erachtet. Auf den ersten Blick scheint es wenig Berührungspunkte zwischen Notfallmedizin und Prävention zu geben, da Notfälle als häufig schwer oder gar nicht vorhersehbare Ereignisse oder Komplikationen gesehen werden. Prävention gründet demgegenüber auf Kenntnis- 
sen über vermeidbare Gesundheitsrisiken und legt Handlungsweisen nahe, die im Gegensatz zur notfallmedizinischen Intervention längere Zeiträume in Anspruch nehmen. Wenn dennoch nach einem engeren Zusammenhang zwischen diesen beiden Phänomenen gefragt wird, so deshalb, weil manche notfallmedizinischen Einsatzdiagnosen nicht-zufällige Häufungen bei bestimmten Personengruppen, an bestimmten Orten bzw. zu bestimmten Zeiten aufweisen. Es ist daher sinnvoll, Risikofaktoren bzw. Risikokonstellationen notfallmedizinischer Komplikationen zu identifizieren und auf ihre präventivmedizinischen Implikationen hin zu überprüfen (Siegrist u. Knesebeck 2005).

Im Zusammenhang mit Prävention tauchen schnell Fragen auf, die eine ethische Auseinandersetzung erforderlich machen. Welche Risiken sind es wert, dass man präventiv tätig wird und auf welcher Grundlage sollte dies entschieden werden? Welchen Nutzen kann eine solche Kampagne haben und wie wird dieser bewertet? Trägt Prävention zu einer Verstärkung gesundheitlicher Ungleichheiten bei und wenn ja, wie kann dies vermieden werden? Mit diesen und weiteren Fragen wird sich der vorliegende Beitrag auseinandersetzen. Im folgenden Kapitel werden zunächst zentrale Konzepte und Strategien der Prävention vorgestellt und aktuelle Kontroversen in der Präventionsforschung skizziert; daraufhin wird auf ethische Aspekte der Prävention eingegangen (s. Kap. 21.3), bevor die Bedeutung der Prävention für die Notfallmedizin am Beispiel von Herz-Kreislauferkrankungen thematisiert wird (s. Kap. 21.4). In Kapitel 21.5 schließlich werden die sich daraus ergebenden ethischen Aspekte in der Prävention von Notfällen diskutiert.

\subsection{Prävention: Definitionen und Konzepte}

Ihrem Grundverständnis folgend will Prävention in erster Linie Krankheitsursachen reduzieren und Krankheitsrisiken vermeiden (Walter et al. 2012). Dem gegenüber steht der Begriff der Gesundheitsförderung, welcher die Förderung von Schutzfaktoren und gesundheitsrelevanten Ressourcen zum Ziel hat (Altgeld u. Kickbusch 2012). Übersetzt man diese Definitionen in Maßnahmen, so wird relativ schnell deutlich, dass eine solche Trennung nicht immer ganz leicht aufrecht zu erhalten ist. Eine ausgewogene Ernährung kann beispielsweise direkt der Gesundheitsförderung zugerechnet werden, trägt jedoch auch zur Vermeidung verschiedener chronischer Erkrankungen und Gesundheitsrisiken bei. Daher wird in diesem Zusammenhang zumeist von Risiko- beziehungsweise Protektivfaktoren gesprochen. Risikofaktoren sind Merkmale, deren Vorhandensein mit einem erhöhten Risiko für Erkrankungen einhergeht. Als Schutzfaktoren lassen sich all diejenigen Faktoren zusammenfassen, die sich positiv auf die Gesundheit auswirken beziehungsweise die negativen Auswirkungen eines Risikofaktors abschwächen oder ganz ausschalten. Eine vitaminreiche Ernährung stärkt die Gesundheit, aufgrund ihrer antioxidativen Wirkung können Vitamine auch dazu beitragen, die negativen Auswirkungen des Rauchens abzumildern.

In der Präventionsforschung wird im Allgemeinen zwischen Primär-, Sekundär- und Tertiärprävention unterschieden. Betrachtet man Prävention auf einem zeitlichen Kontinuum, welches der Entstehung und Entwicklung einer Krankheit folgt, dann würde Primärprävention beim gesunden Menschen ansetzen. Ziel einer primärpräventiven Intervention ist es, die Häufigkeit von Risikofaktoren in einer gesunden 
Population und somit die Entstehung von Neuerkrankungen (Inzidenzen) zu verringern. Maßnahmen der Sekundärprävention haben zum Ziel, die gesundheitlich negativen Auswirkungen von Risikofaktoren zu reduzieren und Krankheiten möglichst früh zu erkennen und zu behandeln. Beispielsweise zählen hierzu Früherkennungsuntersuchungen wie etwa die Mammografie oder das Langzeit-EKC zur Früherkennung von Herz-Kreislauf Erkrankungen. Tertiärprävention möchte hingegen die Verschlimmerung einer bestehenden Erkrankung vermeiden. Klassische Beispiele sind hier rehabilitative Maßnahmen oder edukative Verfahren zum Umgang mit einer Erkrankung. Nun ist es nicht immer ganz einfach, zwischen einer Früherkennung und einer klinischen Manifestation zu unterscheiden. Ist eine sich entwickelnde arterielle Hypertonie ein Risikofaktor für einen Herzinfarkt oder ist sie selbst bereits eine klinisch manifeste Erkrankung? Nicht in jedem Fall lassen sich Sekundär- und Tertiärprävention klar trennen.

Grundsätzlich kann zwischen bevölkerungsbezogenen Präventionsstrategien und einer selektiven Risikogruppenstrategie unterschieden werden (Walter et al. 2012). Letztere bezieht sich auf eine bestimmte eher homogene Gruppe in der Bevölkerung, die möglicherweise bereits einen Risikofaktor trägt. Diese Maßnahmen zeichnen sich häufig durch ihre spezifische Herangehensweise aus, zielen jedoch auf eine zahlenmäßig kleinere Gruppe. Eine Bevölkerungsstrategie hingegen versucht, möglichst breit und möglichst viele Personen zu erreichen. Aufbauend auf der Spezifizierung der Zielgruppe wird dann die Intervention geplant (Leppin 2006).

Eine weitere Unterscheidung bezieht sich darauf, ob Präventionsmaßnahmen am individuellen Verhalten einer Person ansetzen oder an den strukturellen Umweltbedingungen bzw. gesellschaftlichen Verhältnissen, denen die Menschen ausgesetzt sind. $\mathrm{Zu}$ den erstgenannten Maßnahmen der Verhaltensprävention zählen z.B. die ärztliche Beratung zu Risikofaktoren oder zu Lebensstiländerungen, aber auch bevölkerungsbezogene Aufklärungskampagnen oder Maßnahmen zur individuellen Stressbewältigung. Wird jedoch z.B. durch Gesetze oder Regelungen wie etwa dem Rauchverbot in öffentlichen Gebäuden sowie Restaurants und Gaststätten versucht, entsprechende Risikofaktoren zu minimieren, so werden die Maßnahmen der Verhältnisprävention zugerechnet. Denn hier wird eher auf die Verhältnisse abgezielt, in denen bestimmte Verhaltensweisen auftreten oder aber vermieden werden können. Da sich gezeigt hat, dass verhaltenspräventive Maßnahmen allein von begrenzter Reichweite und zum Teil begrenzt wirksam sind (Mühlhauser 2014), wird es in der Prävention als zunehmend wichtig erachtet, die Lebensverhältnisse zu berücksichtigen. So werden die Gruppen mit den größten gesundheitlichen Risiken häufig nicht durch verhaltenspräventive Maßnahmen erreicht (inverse prevention law). Dies sind vor allem die Bevölkerungsgruppen, die über ein vergleichsweise niedriges Einkommen oder eine niedrige Bildung verfügen. Vor diesem Hintergrund ist in aktuellen Studien gezeigt worden, dass die Prävention (insbesondere die Verhaltensprävention) durchaus zu einer Verschärfung gesundheitlicher Ungleichheiten beitragen kann (Janßen et al. 2012; Lorenc et al. 2013).

\subsection{Ethische Aspekte in der Prävention}

Gerade die Hinweise, dass präventive Maßnahmen nicht nur zu einer Verringerung, sondern auch zu einer Verschärfung von sozialen Ungleichheiten in der Gesundheit 
beitragen können, lassen ethische Aspekte der Prävention in den Vordergrund treten (Callahan u. Jennings 2002). Was sind zentrale Annahmen der Prävention und wie werden diese in präventiven Maßnahmen umgesetzt?

Grundsätzlich wird durch eine ethische Auseinandersetzung versucht, auf Fragen Antworten zu finden, die gemäß einer moralischen Wertordnung zufriedenstellend sind. Diese Antworten sollen das Handeln beeinflussen, und zwar in einer Weise, dass dieses als „recht und gut“ bezeichnet werden kann (Laaser 1997). Die Interpretation dessen, was als recht und gut bezeichnet werden kann, mag je nach Wertevorstellungen innerhalb von Gesellschaften und Gruppen variieren (z.B. zwischen Religionen oder unterschiedlichen Berufsständen). Letztlich geht es darum, anhand einer moralisch-ethischen Richtschnur eine entsprechende Handlungsnorm zu begründen, zu entwickeln und anzuwenden. Dies wird als normative Ethik bezeichnet (Strech et al. 2012).

In der utilitaristischen Tradition würde diejenige Maßnahme als richtig und gut bezeichnet werden, die den größten Nutzen bringt. Weniger steht hierbei der individuelle als vielmehr der gesamtgesellschaftliche Nutzen im Vordergrund (Bittner u. Heller 2012). An dieser Stelle werden ethische Konflikte sichtbar, wenn es um die Abwägung individueller Rechte und Freiheiten einerseits und den eher paternalistisch und kommunitaristisch orientierten Ansatz präventiver Interventionen andererseits geht (Callahan u. Jennings 2002; Bayer u. Fairchild 2004). Während in der Bioethik der Medizin der Autonomie und der Freiheit des Individuums der höchste Wert beigemessen wird, lässt sich dies in einer Ethik von Public Health und Prävention so nicht aufrechterhalten (Bayer u. Fairchild 2004). Es sollte demzufolge ein Ziel von Prävention sein, eine ausgewogene Balance zwischen beiden Aspekten zu finden.

Dieser utilitaristischen Position gegenüber stehen vertragstheoretisch orientierte Ansätze, die den Wert von präventiven Handlungen daran bemessen, inwiefern diese auch bei den jeweils schwächsten Mitgliedern der Gesellschaft ansetzen und deren gesundheitlichen Risiken mindern. Diese Ansätze beruhen auf der Grundannahme, dass jedem Individuum das gleiche Maß an Freiheiten zugesprochen wird, solange dieses auch mit gleichen Freiheiten für Andere vereinbar ist (Bittner u. Heller 2012). Alle Mitglieder schließen dieser Theorie folgend einen hypothetischen, jedoch allgemeingültigen Vertrag über Gerechtigkeitsprinzipien, anhand welcher Freiheiten definiert werden können. Dazu kommt das sogenannte Differenzprinzip, wonach Ungleichheiten nur bestehen dürfen, wenn sie in anderen möglichen Ordnungsprinzipien stärker ausfallen würden. Daher würde hier nicht unbedingt diejenige präventive Intervention gewählt, die den größten gesamtgesellschaftlichen Nutzen bringt, sondern vielmehr diejenige, die unter Beachtung der Freiheiten Aller insbesondere die am schwächsten gestellten Individuen stärkt. Unklar bleibt in diesem Ansatz, ob es tatsächlich möglich ist, unter idealen Bedingungen Gerechtigkeitsprinzipien zu definieren und inwiefern nicht schon Machtpositionen in einem solchen Aushandlungsprozess wirksam sind.

Dem dritten klassischen Prinzip, der libertären Konzeption von Gerechtigkeit, liegt das Grundrecht auf Eigentum als oberstes Ordnungsprinzip zugrunde, dem Recht also, über rechtmäßig erworbenes Eigentum selbst zu bestimmen. Demzufolge lässt sich Prävention bewerten, wenn sie allen gemäß ihrer Menge an Eigentümern freien Zugang zu diesen Interventionen gewährt. Allerdings ist dieser Ansatz kritisch be- 
trachtet worden, da sich die Annahme der Rechtmäßigkeit von Eigentum nicht ohne weiteres aufrechterhalten lässt.

Keine der genannten ethischen Grundausrichtungen konnte sich in den Gesundheitswissenschaften allgemeingültig durchsetzen. Vielmehr lässt sich eine praktische Ethik für den Bereich der Prävention am ehesten aus einem kohärentistischen Verfahren heraus entwickeln. Hier wird nicht auf ein einziges, letztgültiges moralisches Prinzip verwiesen, sondern auf mehrere konsensfähige Prinzipien innerhalb einer bestimmten Gesellschaft. Beispielsweise haben sich als solche Grundprinzipien in der Medizin das Prinzip des Nichtschadens, das Wohl des Patienten, der Respekt der Autonomie sowie die Gerechtigkeit etabliert (Beauchamp u. Childress 2008).

Für eine ethische Bewertung von präventiven Maßnahmen ist ein Instrumentarium notwendig, welches Orientierung und Regeln bietet. Strech und Kollegen (2012) haben ein solches Instrumentarium entwickelt, das auf sechs Kriterien beruht:

1. Beurteilung des Nutzenpotenzials,

2. Abschätzung des Schadenspotenzials,

3. Respekt der Autonomie,

4. Gerechtigkeit,

5. Effizienz und

6. Legitimität.

An erster Stelle steht die Bewertung des Nutzenpotenzials einer präventiven Maßnahme. Hierbei geht es um die Fragen der Interventionsziele genauso wie die Bedeutung der Maßnahme für die jeweilige Zielpopulation. Auch sollte die Evidenz für diesen potenziellen Nutzen geprüft werden, zudem sollte diese möglichst transparent und offen dargelegt werden. Direkt an den Nutzen schließt die Frage nach möglichem Schaden als zweites Prinzip an. Gehen aus der Intervention bestimmte Risiken oder Belastungen hervor und wie sind diese einzuschätzen? Im Sinne des Empowerment sollte jede Maßnahme zu einer Förderung der Gesundheitskompetenz und der Selbstbestimmung in der Zielpopulation führen, wie im dritten Kriterium gefordert wird. Dieses beinhaltet im Rahmen eines informed consent freie Entscheidungen über die Teilnahme und Nichtteilnahme sowie die freie Abwägung von Nutzen und Schaden einer Intervention. Als viertes Kriterium wird die Gerechtigkeit genannt, wobei insbesondere Zugangsbarrieren und Zielgruppenspezifität im Vordergrund stehen, ohne dabei aber mögliche Labeling-Effekte außer Acht zu lassen. Letzteres meint, dass gerade bei spezifischen Kampagnen die Wahrscheinlichkeit erhöht ist, dass der Zielgruppe einer solchen Kampagne entsprechende Eigenschaften zugeschrieben werden, welche im Rahmen der Intervention transportiert werden. Grundlegend wird hier auch danach gefragt, ob die jeweilige Intervention allen gesellschaftlichen Subgruppen gleichermaßen zugute kommt oder ob Ungleichheiten bestehen oder gar verstärkt werden (Bittner u. Heller 2012). Gesamtgesellschaftlich stellt sich, insbesondere unter Verwendung öffentlicher Mittel, die Frage nach einem Kosten-NutzenVerhältnis und dem sinnvollen Gebrauch von Mitteln. Dieses fünfte Kriterium beinhaltet ebenfalls die Frage, inwiefern mögliche Alternativen geprüft worden sind. Abschließend wird der Entscheidungsprozess einer Prüfung unterzogen, inwiefern dieser legitimiert, transparent, unter Offenlegung der Entscheidungsbegründung sowie mit möglichst hohem Grad an Partizipation durchlaufen wurde. Die Entscheidung selbst sollte ein reguliertes Verfahren durchlaufen, dessen Regeln auf einem gemeinsamen Konsens beruhen. 
Es bietet sich an, präventive Maßnahmen anhand solcher Kriterien zu planen und zu evaluieren. Viele dieser Kriterien (Gerechtigkeit, Empowerment, Kosten-Nutzen Relation, Legitimität) finden sich z.B. im Kooperationsverbund „gesundheitliche Chancengleichheit“ (www.gesundheitliche-chancengleichheit.de/praxisdatenbank). Die im Rahmen dieses Verbundes entwickelte Praxisdatenbank bietet eine große bundesweite Übersicht über Angebote und Maßnahmen der Gesundheitsförderung und Prävention, die sich insbesondere an Menschen in schwieriger sozialer Lage richten. Wichtiges Ziel des Verbundes ist es zudem, die Qualität in der Prävention und Gesundheitsförderung sozial Benachteiligter zu erfassen und zu verbessern, was anhand von Good-Practice-Kriterien in die Tat umgesetzt wird.

\subsection{Prävention in der Notfallmedizin}

Um gesundheitliche Risiken im Sinne einer Prävention zu minimieren, ist es notwendig, bestimmte Verteilungsmuster und Häufigkeiten in der Bevölkerung zu identifizieren. Welche Muster ergeben sich bei der Betrachtung von medizinischen Notfällen in Deutschland? Zunächst lässt sich feststellen, dass knapp 40\% aller Krankenhauspatienten in Deutschland als medizinische Notfälle eingeliefert werden. Als Hauptursachen für medizinische Notfälle gelten neben Verletzungen und Vergiftungen insbesondere Erkrankungen des Herz-Kreislauf Systems, wie etwa Herzinsuffizienz, Myokardinfarkt oder Schlaganfall (Destatis 2014). Insbesondere der „plötzliche Herztod" stellt nach wie vor eine große Herausforderung in der Notfallmedizin dar. Zwar ist die Koronarsterblichkeit in den letzten Jahren rückläufig, Rückgänge der spezifischen Mortalität um bis zu 50\% in den letzten 30 Jahren konnten gezeigt werden, jedoch ist die Rate der prähospital Verstorbenen nach wie vor hoch (GBE Bund 2013). So zeigen Daten der KORA Studie, dass knapp 40\% der Herzinfarkt-Patienten vor Erreichen des Krankenhauses versterben (Löwel et al. 2005).

Gerade Erkrankungen des Herz-Kreislauf-Systems weisen einige typische soziodemographische Verteilungsmuster auf. So sind häufiger Männer, ältere Menschen und Personen mit einem niedrigen sozioökonomischen Status betroffen (RKI 2014). Zudem weist das Merkmal der langen symptomlosen Entwicklungsphase zahlreicher Herz-Kreislauferkrankungen auf Potenziale der Prävention hin. Es wurde bereits eine ganze Reihe von biomedizinischen, verhaltensbezogenen und psychosozialen Risikofaktoren für Herz-Kreislauferkrankungen identifiziert, welche zum Ziel präventiver Maßnahmen wurden. Im Hinblick auf verhaltensbezogene Faktoren wird häufig auf die weitere Verringerung von Rauchprävalenz sowie auf die Förderung von körperlicher Bewegung oder gesunder Ernährung verwiesen. Eine Vielzahl von Programmen widmet sich diesem Feld. Dies wird insbesondere durch aktuelle Programme wie die Europäische Charter zur Herzgesundheit (European Heart Health Charter, www.heartcharter.org) sowie die Leitlinie der Europäischen Gesellschaft für Kardiologie zur Prävention von Herz-Kreislauf-Erkrankungen deutlich. Der Prävention von Herzerkrankungen wird auch von Seiten der Akteure im Gesundheitswesen eine wichtige Rolle zugeschrieben und Anstrengungen werden unternommen, die vorgeschlagenen Maßnahmen auch langfristig zu implementieren, wie beispielsweise in der ärztlichen Beratung von Risiken (www.arriba-hausarzt.de, www.heartscore.org).

Präventive Maßnahmen im Rahmen der Notfallmedizin sollten insbesondere dazu beitragen, die nach wie vor hohe Anzahl an Patienten, die noch vor Erreichen des 
Krankenhauses versterben, zu verringern. Hierbei könnte die Identifizierung von Patienten mit besonders hohen Risiken hilfreich sein, wenn Patienten mit ersten Symptomen und Beschwerden notärztliche Hilfe in Anspruch nehmen. Zur Identifizierung von Risikogruppen sowie zur Einschätzung individueller Risiken wurden Risikoprofile entwickelt. Als Gruppe mit besonders hohen Risiken für einen Herzinfarkt (>10\%) in den nächsten 10 Jahren werden diejenigen Personen identifiziert, welche bereits mit diagnostizierter KHK oder anderen manifesten Erkrankungen an den Herzkranzgefäßen leben (Stenosen, periphere arterielle Verschlusskrankheit etc.). Auch liegen zur Identifizierung individueller Risikoprofile länderspezifische Tests vor (wie z.B. Systematic Coronary Risk Evaluation SCORE, De Backer 2003). Gezielte Aufklärung und Interventionen könnten hier zukünftig zu einer verbesserten notfallmedizinischen Behandlung beitragen. So würden Patienten und ihre Angehörigen besser in die Lage versetzt, erste Symptome rechtzeitig wahrzunehmen und die ersten notfallmedizinischen Schritte zeitnah einzuleiten. Zwar konnte gezeigt werden, dass die Symptomwahrnehmung in Deutschland insbesondere im Vergleich zu anderen europäischen Ländern relativ hoch ist. Jedoch besteht nach wie vor großes Potenzial für Aufklärung und Information. Von den zentralen Anzeichen für einen Herzinfarkt wurde nur der Brustschmerz von der Mehrheit der Befragten angegeben, kein einziges Anzeichen für einen Schlaganfall wurde von mehr als 50\% der Befragten korrekt genannt (Mata et al. 2012). Auch würde nur knapp die Hälfte der Studienteilnehmer bei Wahrnehmung dieser Symptome sofort einen Notruf absetzen. Zudem ist das Wissen um Symptome der KHK nach soziodemographischen Merkmalen ungleich verteilt, etwa nach sozialer Schichtzugehörigkeit, Alter und Geschlecht. Ältere Personen, Männer sowie Personen mit geringem Bildungsstand sind in der Regel schlechter informiert. Dementsprechend sollten die jeweiligen Angebote möglichst niedrigschwellig und zielgruppenspezifisch ausgerichtet sein. An dieser Stufe setzt auch das PREMISE Programm der WHO an. Ziel des Programmes ist die Verbesserung der Gesundheitsversorgung von Menschen mit diagnostizierter KHK. Hierbei steht insbesondere die Nachsorge durch Hausärzte und andere Hilfsdienste in der Community im Vordergrund, eine engere Vernetzung kann dabei dazu beitragen, dass im Notfall Hilfe zur Verfügung steht (WHO 2015).

Hoffnungen wurden auch auf die von Laien zu bedienenden Defibrillatoren gesetzt, welche mehr und mehr im öffentlichen Raum zur Verfügung gestellt werden. Jedoch spricht gegen einen Beitrag dieser Defibrillatoren zu einer bedeutenden Senkung der Letalität, dass sich die große Mehrheit der Ereignisse bei Patienten zu Hause ereignet. Zu einer Verringerung der Risiken können gemäß der aktuellen Leitlinie der Deutschen Gesellschaft für Kardiologie (DGK) zur Prävention von Herz-Kreislauf Erkrankungen auch Screenings für Männer $\mathrm{ab} 40$ Jahren und für Frauen ab 5 o beitragen. Dies kann zu einer besseren Früherkennung und zu einer Vermeidung von Komplikationen und Notfällen beitragen und wird entsprechend von der DGK empfohlen. Jedoch sind solche Früherkennungsuntersuchungen nicht unumstritten, da sie stets auch die Möglichkeit von Fehldiagnosen und Überversorgung mit einschließen und mit entsprechenden Risiken für die Teilnehmer behaftet sind (Mühlhauser 2014).

Neben einer Aufklärung zu Symptomen und zu verbessertem Umgang mit Krankheiten des Herz-Kreislauf Systems sollten auch die Anstrengungen zu Maßnahmen der Ersten Hilfe in der Bevölkerung ausgeweitet werden. Dies beinhaltet ein breiteres Angebot an Kursen, welches deutlich über den Bereich der betrieblichen Gesundheitsversorgung hinausgehen sollte. Auch sollten die Kosten einer Teilnahme mög- 
lichst gering gehalten werden, um so gleiche Zugangschancen zu gewährleisten. Als Beispiel sind an anderer Stelle bereits wohnortsnahe Ausbildungsinitiativen genannt worden (Siegrist u. Knesebeck 2005). Zwar sind im Rahmen der Führerscheinprüfungen auch Kurse der Ersten Hilfe für alle verpflichtend, jedoch liegen diese Kurse für die meisten Personen bereits jahrelang zurück. Entsprechend ernüchternd sind auch Zahlen zur Einleitung von Reanimationsmaßnahmen in Deutschland, wonach in nur knapp 15\% aller registrierten Fälle von plötzlichem Herztod die Reanimation durch Anwesende vor Eintreffen des Rettungsdienstes begonnen wurde. Die Quoten unterscheiden sich je nach Ort des Geschehens, wobei am Arbeitsplatz die höchste Wahrscheinlichkeit besteht, dass mit einer Reanimation begonnen wird (Gräsner et al. 2012). Auch trauen sich viele Laien die Reanimation nicht $\mathrm{zu}$, möglicherweise aus Angst vor Fehlern oder rechtlichen Konsequenzen. Eine Studie aus den Niederlanden konnte zeigen, dass eine verbesserte Laienausbildung in Reanimationsmaßnahmen einen positiven Einfluss auf die 30-Tage Überlebensrate nach einem Herzinfarktereignis hat (Waalewijn et al. 2001). Insgesamt ist die Datenlage in diesem Bereich als eher schwach zu bezeichnen. Verschiedene Initiativen zur Sensibilisierung der Bevölkerung für Erste-Hilfe-Maßnahmen sind international bekannt, wie beispielsweise das Programm der American Heart Association „Emergency Cardiovascular Care $(E C C)^{\prime \prime}$. Gerade weil Erste Hilfe zu einer Senkung der Letalität akuter Herzkreislauferkrankungen und zu einer Verminderung von Folgeschäden sowie zu einer Verbesserung der Schnittstelle zwischen Laienhilfe und dem professionellen Notfallsystem beitragen kann, sollte der Ausbau bestehender Programme und die Verbesserung des Kenntnisstands in der Bevölkerung vorangetrieben werden.

\subsection{Ethische Aspekte in der Prävention von Notfällen}

Wie im vorangegangenen Kapitel skizziert, richten sich Maßnahmen zur Prävention lebensbedrohlicher Komplikationen von Herz-Kreislauferkrankungen vor allem auf eine verbesserte Früherkennung und eine darauf aufbauende gezielte Beeinflussung von Risikofaktoren sowie auf bevölkerungsweite Aufklärungs- und Schulungsprogramme. Ihre vordringlichen Ziele sind eine Verbesserung des Kenntnisstandes über Art und Schweregrad von Manifestationsformen des akuten Myokardinfarkts und des Schlaganfalls, eine Verkürzung des Zeitfensters von der Notfallmanifestation bis zum Eintreffen notärztlicher Hilfe und ein intensiviertes Training von Maßnahmen Erster Hilfe bei Betroffenen und ihren Angehörigen (Siegrist u. Knesebeck 2005). In diesem abschließenden Kapitel soll versucht werden, die in Kapitel 21.3 beschriebenen ethischen Kriterien (Beurteilung des Nutzen- und Schadenspotenzials, Respekt der Autonomie, Gerechtigkeit, Effizienz und Legitimität; Strech et al. 2012) exemplarisch auf diese präventiven Maßnahmen zu beziehen.

Aussagekräftige Ergebnisse zur Nutzen-Schaden-Bewertung stammen üblicherweise aus randomisiert-kontrollierten Studien. Allgemein gesprochen besteht in Bezug auf entsprechende Studien zur Wirksamkeit von präventiven Maßnahmen ein deutliches Defizit; dies gilt auch für Maßnahmen zur Prävention von Notfällen. Im Hinblick auf sekundärpräventive Früherkennungsuntersuchungen sprechen die Befunde eher für eine Überschätzung des Nutzens und eine Unterschätzung des Schadens (Mühlhauser 2014). Seit einigen Jahren besteht eine Cochrane Gruppe für Public Health, die durch Veröffentlichung von Reviews zur Evidenzbasierung von Prävention beitragen will 
(http://ph.cochrane.org/de/home). Aufklärungs- und Schulungsprogramme zu Risikofaktoren und Manifestationsformen von Herz-Kreislauferkrankungen wie auch das Training von Maßnahmen Erster Hilfe zielen auf die Stärkung von Gesundheitskompetenz und tragen so zu einem Empowerment der Zielgruppe bei. Zum ethischen Prinzip des Respekts der Autonomie gehört darüber hinaus eine verständliche Information über das Nutzen- und Schadenspotenzial der präventiven Maßnahmen, damit potenzielle Teilnehmer eine informierte Zustimmung geben können. Gerade bei Früherkennungsuntersuchungen sind die entsprechenden Informationen häufig komplex und werden z.T. missverständlich oder gar irreführend aufbereitet und zur Verfügung gestellt. Inzwischen gibt es in Deutschland mehrere Initiativen, die sich der verständlichen Aufbereitung von entsprechenden Gesundheitsinformationen verschrieben haben (www.gesundheitsinformation.de/; www.harding-center.mpg. de/de). Im Hinblick auf das Kriterium der Gerechtigkeit hat sich wiederholt gezeigt, dass bevölkerungsweite Aufklärungs- und Schulungsprogramme wie auch andere Interventionen, die auf das individuelle Verhalten zielen, eher zu einer Ausweitung als zu einer Reduktion von gesundheitlichen Ungleichheiten beitragen (Lorenc et al. 2013). Auch werden Früherkennungsuntersuchungen von Menschen mit einem niedrigen sozialen Status seltener in Anspruch genommen (Klein et al. 2014). Insofern ist auch bei Präventionsmaßnahmen in der Notfallmedizin verstärkt auf das Kriterium der Gerechtigkeit zu achten, z.B. indem häufiger verhältnispräventive Ansätze integriert werden und präventive Maßnahmen entsprechend zielgruppenspezifisch entwickelt werden. Ähnlich wie bei der Wirksamkeit besteht im Bereich der Effizienz von präventiven Maßnahmen ein erheblicher Forschungsbedarf. Es mangelt hier nach wie vor an belastbaren Daten. Das letzte Kriterium der Legitimität besagt, dass präventive Maßnahmen durch eine legitimierte Instanz nach einem transparenten und fairen Entscheidungsprozess implementiert werden sollen. Dabei spielt die Partizipation von betroffenen Populationen eine wichtige Rolle. Auch bei der Gestaltung von Maßnahmen zur Prävention von Notfällen ist es wichtig, die Zielgruppe (z.B. Menschen mit erhöhtem Myokardinfarktrisiko) zu befähigen, ihre eigenen Bedürfnisse zu formulieren und Wünsche, Ideen und Vorstellungen bei der Planung, Umsetzung und Durchführung der präventiven Maßnahmen einzubringen.

\section{Literatur}

Altgeld T, Kickbusch I (2012) Gesundheitsförderung. In: Schwartz FW, Walter U, Siegrist I, Kolip P, Leidl R, Dierks M-L, Busse R, Schneider N (Hrsg.) Public Health: Gesundheit und Gesundheitswesen. Urban \& Fischer, München, S. 187-196

Bayer R, Fairchild AL (2004) The genesis of public health ethics. Bioethics 18(6): 473-492

Beauchamp TL, Childress JF (2008) Principles of biomedical ethics. Oxford University Press, New York.

Bittner R, Heller S (2012) Ethik in den Gesundheitswissenschaften. In: Hurrelmann K, Laaser U (Hrsg.) Handbuch Gesundheitswissenschaften (vollst. überarb. Auflage). Beltz Juventa, Weinheim, S. 221-236

Callahan D, Jennings B (2002) Ethics and Public Health: forging a strong relationship. Am I Pub Health 92(2): 169-176

Destatis Statistisches Bundesamt (2014) Zahl der Woche vom 18. Februar 2014. www.destatis.de (Zugriff am 15.05.2015)

Deutsche Krankenhausgesellschaft (DKGEV) (2015) Diagnosedaten der Patientinnen und Patienten in Krankenhäusern 2013. http://www.dkgev.de/dkg.php/cat/62/title/Statistik (Zugriff am 15.05.2015)

Gesundheitsberichterstattung des Bundes (GBE) (2013) Daten zu Herzinfarkten in der Region Augsburg (Mortalität, Morbidität, Letalität, Vorerkrankungen, medizinische Versorgung). www.gbe-bund.de (Zugriff am 15.05.2015) 
Go AS, Bauman MA, Coleman King SM Fonarow GC, Lawrence W, Williams KA, Sanchez E (2014) An Effective Approach to High Blood Pressure Control - A Science Advisory From the American Heart Association, the American College of Cardiology, and the Centers for Disease Control and Prevention. I Am Coll Cardiol 63: 1230-1238

Gräsner et al. 2012

Janßen C, Sauter S, Kowalski C (2012) The influence of social determinants on the use of prevention and health promotion services: results of a systematic literature review. Psychosoc Med 9: DOC07

Klein I, Hofreuter-Gätgens K, Knesebeck 0 vd (2014) Socioeconomic status and the utilization of health services in Germany: a systematic review. In: Janßen C, Swart E, von Lengerke T (Hrsg.) Health care utilization in Germany: Theory, Methodology, and Results. Springer, New York, S. 117-143

Laaser U (1997) Ethische Fragen in der Prävention. In: Allhoff P, Flatten G, Laaser U (Hrsg.) Krankheitsverhütung und Früherkennung - Handbuch der Prävention. Springer Verlag, Berlin, S. 76-86

Leppin A (2006) Zielgruppenspezifische Prävention. In: Haisch J, Hurrelmann K, Klotz T (Hrsg.) Medizinische Gesundheitsförderung und Prävention. Verlag Hans Huber, Bern, S. 17-24

Leppin A (2010) Konzepte und Strategien der Prävention. In: Hurrelmann K, Klotz T, Haisch I (Hrsg.) Prävention und Gesundheitsförderung. Verlag Hans Huber, Bern, S. 35-45

Lorenc T, Petticrew M, Welch V, Tugwell P (2013) What types of interventions generate inequalities? Evidence from systematic reviews. I Epidemiol Community Health 67 (2): 288-290

Löwel H, Meisinger C, Heier M, Hörmann A, Scheidt W von (2005) Herzinfarkt und koronare Sterblichkeit in Süddeutschland. Dtsch Arzteblatt 103(10): A-616

Mata I, Frank R, Gigerenzer G (2012) Symptom recognition of heart attack and stroke in nine european countries: a representative study. Health Expect 17: 376-387

Minkler M, Wilson N, Wallerstein NB (2008) Improving health through community organization and community building. In: Glanz K, Rimer BK, Viswanath K (Hrsg.) Health behavior and health education: theory, research and practice. Jossey Bass, San Francisco, S. 287-312

Mühlhauser I (2014) Zur Überschätzung des Nutzens von Prävention. Z Evid Fortbild Qual Gesundh ZEFQ 108 (4): 208-218

Robert Koch-Institut RKI (2014) Daten und Fakten: Ergebnisse der Studie „Gesundheit in Deutschland aktuell 2012". Beiträge zur Gesundheitsberichterstattung des Bundes. RKI, Berlin, S. 72-74

Siegrist I, Knesebeck 0 vd (2005) Akutmedizin und Prävention. In: Madler C, Jauch K-W, Werdan K, Siegrist J, Pajonk F-G (Hrsg.) Das NAW-Buch - Akutmedizin der ersten 24 Stunden. Urban \& Fischer, München, S. 1217-1225

Strech D, Neitzke G, Marckmann G (2012) Public-Health-Ethik: normative Grundlagen und methodisches Vorgehen. In: Schwartz FW, Walter U, Siegrist J, Kolip P, Leidl R, Dierks M-L, Busse R, Schneider N (Hrsg.) Public Health: Gesundheit und Gesundheitswesen. Urban \& Fischer, München, S. 136-143

Waalewijn RA, Tijssen JG, Koster RW (2001) Bystander initiated actions in out-of-hospital cardiopulmonary resuscitation: results from the Amsterdam Resuscitation Study (ARRESUST). Resuscitation 50: 273-279

Walter U, Robra BP Schwarf FW (2012) Prävention. In: Schwartz FW, Walter U, Siegrist J, Kolip P, Leidl R, Dierks M-L, Busse R, Schneider N (Hrsg.) Public Health: Gesundheit und Gesundheitswesen. Urban \& Fischer, München, S. $196-223$

WHO (2015) Prevention of recurrences of myocardial infarction and stroke study - the PREMISE programme. www. who.int/cardiovascular_diseases/priorities/secondary_prevention/country/en/ (Zugriff am 19.08.2015)

\section{Internetquellen}

Cochrane Public Health Group: http://ph.cochrane.org/de/home

European Heart Health Charter: www.heartcharter.org

Gesundheitsinformationen des IQWiG: www.gesundheitsinformation.de/

Harding Zentrum für Risikokompetenz: www.harding-center.mpg.de/de

Instrumente zur Ermittlung und Beratung von Risikofaktoren der KHK: www.arriba-hausarzt.de,

www.heartscore.org

Kooperationsverbund Gesundheitliche Chancengleichheit: www.gesundheitliche-chancengleichheit.de/praxisdatenbank 


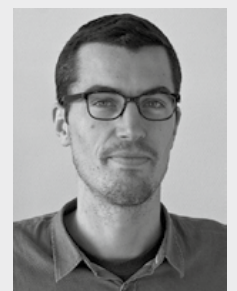

\section{Dr. phil. Nico Vonneilich}

Studium der Soziologie, Psychologie und Politikwissenschaften an den Universitäten Münster und Hamburg. Seit 2008 wissenschaftlicher Mitarbeiter am Institut für Medizinische Soziologie des Universitätsklinikums Hamburg-Eppendorf (UKE). Forschungsschwerpunkte sind insbesondere soziale Einflüsse auf Gesundheit und Krankheit und ihre möglichen Erklärungen.

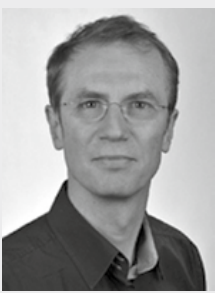

Prof. Dr. phil. Olaf von dem Knesebeck

Studium der Soziologie und Psychologie in Düsseldorf, Magister Artium 1993, Promotion 1997, Habilitation 2003. Seit 2004 Professor für Medizinische Soziologie, Direktor des Instituts für Medizinische Soziologie am Universitätsklinikum Hamburg-Eppendorf. Arbeitsschwerpunkte: Soziale Einflüsse auf Gesundheit und Versorgung, internationale Vergleiche in der Sozialepidemiologie und Versorgungsforschung. 